\title{
Radiation-Induced Breast Angiosarcoma After Breast Cancer: A Case Report
}

\author{
Rodríguez-Fernández V, Cameselle-Cortizo L, García-Mallo A, Novo A, Cameselle-Teijeiro JF and \\ Schmitt FC \\ ${ }^{1}$ Clinical Oncology Research Center ADICAM. Cangas, Spain \\ ${ }^{2}$ Service of Obstetrics and Gynecology. Álvaro Cunqueiro Hospital. Vigo, Spain \\ ${ }^{3}$ Breast Pathology Unit. Meixoeiro Hospital. Vigo. Spain \\ ${ }^{4}$ Professor of Obstetrics and Gynecology at the Faculty of Medicine. University of Santiago de Compostela. Santiago de Compostela, Spain \\ ${ }^{5}$ Professor at the Faculty of Medicine. University of Porto. Porto, Portugal \\ *Corresponding author: Rodríguez Fernández V, Service of Obstetrics and Gynecology, Vigo University Hospital Complex, Álvaro Cun- \\ queiro Hospital, Spain
}

To Cite This Article: Rodríguez Fernández V. Radiation-Induced Breast Angiosarcoma After Breast Cancer: A Case Report. 2020 - 10(3). AJBSR. MS.ID.001506. DOI: 10.34297/AJBSR.2020.10.001506.

Received: 眥 July 15, 2020; Published: 嗢 September 16, 2020

\begin{abstract}
Radiation-induced breast angiosarcoma is a rare but very aggressive tumor, with high local recurrence rate and poor survival. The approximate incidence of this tumor after Radiation Therapy (RT) for Breast Cancer (BC) is $0.1 \%$. The clinical presentation and diagnostic imaging are nonspecific; so angiosarcomas are often initially confused with dermatoses attributable to RT, leading to delays in diagnosis. In this article we describe the clinical, radiological and pathological findings of a 76-year-old woman who developed an angiosarcoma two and a half years after RT for BC and died 11 months after this diagnosis. It represents a clinical, radiological and histological challenge. We must always consider and rule out this entity in the face of radioinduced vascular lesions, since early diagnosis and aggressive treatment with free margins are the main prognostic factors.
\end{abstract}

\section{Introduction}

Breast sarcomas are heterogeneous tumors that arise from mesenchymal cells, from the mammary connective tissue. They are rare, representing less than $1 \%$ of all breast cancers (BC) and less than $5 \%$ of all soft tissue sarcomas [1]. The subtype of sarcoma strongly linked to the breast is the angiosarcoma, originating in the endothelial vascular or lymphatic cells.

Breast angiosarcoma is an aggressive subtype, with rapid proliferation and infiltration of surrounding tissues; generally associated with poor prognosis. Based on their etiology, it can be classified as primary (de novo) and secondary (related to BC treatment: after radiation therapy -RT-, or due to lymphedema in the arm or breast/chest wall after surgical treatment) $[2,3]$.
The use of adjuvant RT is well established in the treatment of BC. It has been shown in multiple large clinical trials to improve loco-regional control and survival rates [4].

Radiation-induced angiosarcoma after BC is a rare entity that complicates approximately $0.1 \%$ of all BC treated with RT $[5,6]$ and is associated with a poor prognosis.

The first description of a bone sarcoma after RT for BC was reported by Beck in 1922 [7]. Warren and Sommer in 1936 [8] reported a case of breast angiosarcoma. In 1948, Cahan et al. [9] defined the criteria for the diagnosis of radiation-induced sarcoma: a location in the anterior field of RT, a latency period of at least 3 years, and a histological discordance between primary and secondary tumor. 
In this report, we present a case of radio-induced breast angiosarcoma after breast conserving surgery and adjuvant RT in a patient with BC; an infrequent entity with difficult diagnosis and poor prognosis.

\section{Case Report}

We present the case of a woman diagnosed with $\mathrm{BC}$ at the age of 73. She has no known family history of cancer. Her medical history includes: menarche at the age of 16 , menopause at 48,4 previous deliveries and first birth at the age of 31. Diagnosed several years ago with coronary insufficiency, with episodes of angina pectoris and arterial hypertension.

She consulted for a nodule in the left breast of two months of evolution. On clinical examination, a nodule of approximately $5 \times 3$ $\mathrm{cm}$ was palpated in the upper external quadrant, adhered to the skin and not to deep planes; the armpit was negative on palpation. Mammography revealed two masses of 2 and $1 \mathrm{~cm}$, highly suspicious of malignancy (BI-RADS 5) (Figure 1).
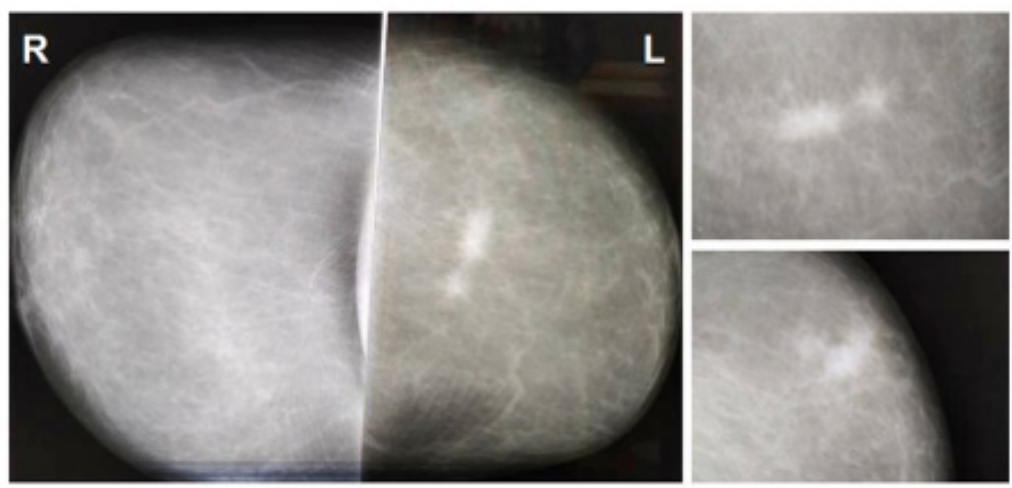

Figure 1: Mammography (BI-RADS 5): Two nodular formations of 2 and $1 \mathrm{~cm}$, very close to each other; with an indefinite contour, irregular shape, high density and surrounded by a peritumoral halo.
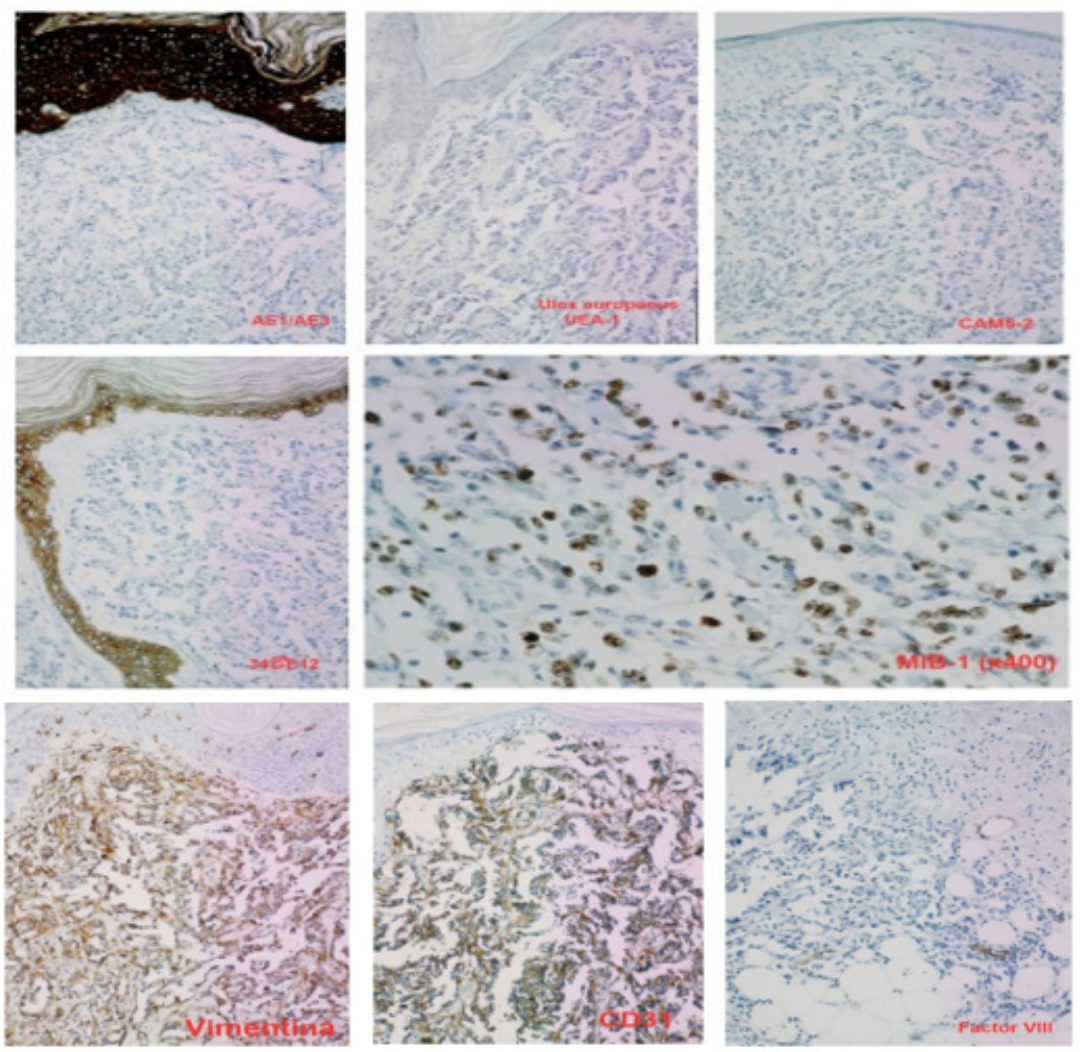

Figure 2: Immunohistochemistry showed: negativity for AE1/AE3, UEA-1, CAM5.2 and 34ßE12; a cell proliferation index (MIB-1) of 70-80\%; positivity for Vimentin, CD31 and focally for Factor VIII-related Antigen. 
The surgery was limited to a quadrantectomy of the left breast, without axillary lymphadenectomy due to complications with anesthesia (cardiac arrhythmia) that forced the surgery to be interrupted. Histological examination confirmed the presence of an Invasive Breast Carcinoma of No Special Type (NST) with a maximum diameter of $4 \mathrm{~cm}$ and infiltration of the surgical margins. The post-surgical extension study was negative for metastasis. The treatment was completed with RT: 50 Gy/25 sessions on the left breast, using two tangential fields (internal and external) and $50 \mathrm{~Gy} / 25$ sessions on the left supraclavicular and axillary lymph node areas, using two shaped fields (anterior and posterior).

Two years after surgery, a skin retraction was observed at the scar level, which was reported in a biopsy as scar tissue. The follow-up mammography, 6 months after the skin retraction biopsy, showed no inflammatory or malignancy pathology. However, two months later, in the area of the surgical scar, a wine-red skin plate appeared with slow but progressive growth. Four months after its appearance, a biopsy of the lesion was performed, which was reported as suggestive of $\mathrm{BC}$ recurrence. Performing a simple mastectomy with axillary lymphadenectomy, allowed us to correct the error and confirm the diagnosis of poorly differentiated (grade III) angiosarcoma, located in the scar of the previous lumpectomy. Five axillary lymph nodes that were negative for malignancy were isolated. The immunohistochemical study showed that the tumor cells were negative for hormone receptors (estrogens and progesterone); they were also negative for low and high molecular weight cytokeratins (CAM5.2, AE1/AE3 and 34ßE12), protein p53, CD34 and Ulexeuropaeus (UEA-1). However, the angiosarcoma cells were positive for Vimentin, CD31, and especially for Factor VIII-related antigen. The cell proliferation index (MIB-1) was 70-80\% (Figure 2).

Six months after mastectomy, a new recurrence was observed on the surgical scar. The bone and liver scan did not show suspicious images of spreading of the disease. The histological study confirmed that the reddish skin nodule infiltrated the striated muscle and affected the deep surgical margin. Finally, our patient died 11 months after the initial diagnosis of angiosarcoma.

\section{Discussion}

The age of onset of secondary angiosarcomas is greater than that of primary angiosarcomas. The average age at diagnosis for primary angiosarcomas is less than 40 years in most series (between 20 and 40 years) [2,10]. However, the average age for secondary angiosarcomas is generally greater than 60 years (range between 40 and 90 years) $[6,11,12]$ and they also appear in patients who have received treatment for $\mathrm{BC}$. The age of presentation of our patient coincides with the range reported in the literature.

Radiation-induced angiosarcomas follow the criteria established by Cahan et al. in 1948 [9], which were modified by Arlen et al. in 1971 [13]: a location in the previous RT field, a latency period of at least 3 years (period ranging from 1 to 26 years in the literature) $[6,11,12,14,15]$ and a histological discordance between primary and secondary tumor. In our case, the patient follows all these criteria.

One of the risks associated with the use of RT is the development of secondary malignancies, which was a debate when breast conserving treatment for BC emerged. Huang et al. [16] revealed a 15.9-fold increased risk of developing angiosarcomas for BC patients treated with RT. RT can increase the risk by causing radiogenetic mutations in the irradiated field and also by contributing to the development of lymphedema. Radiation-induced angiosarcoma is a rare but serious complication of RT. However, the risk of developing angiosarcoma does not outweigh the benefit of the treatment [17].

The incidence of radiation-associated angiosarcoma does not appear to be influenced by the type of surgery performed, but there appears to be an interaction between RT and lymphedema [18]. In addition to improvement in the area of RT, the increased use of conservative surgery with selective sentinel node biopsy has decreased the incidence of treatment-associated lymphedema, decreasing the risk of secondary angiosarcoma.

Most studies support a relationship between RT dose and risk of radiation-induced sarcoma; it is rarely seen after low doses $(<40$ Gy), with a minimum dose of 10 Gy for its development [18]. As an example, in a cohort of 6597 BC patients, of whom 14 developed posterior sarcoma: compared to doses $<14 \mathrm{~Gy}$, the odds ratio for sarcoma in women receiving 14-44 Gy was 1.6 and 30.6 for doses> 44 Gy [19].

Kirova et al. [5] demonstrated an incidence of radiation-induced sarcoma in BC patients of $0.07 \%$ at 5 years, $0.27 \%$ at 10 years and $0.48 \%$ at 15 years, confirming the importance of longterm follow-up. In a recent study, Rombouts et al. estimated a risk of radiation-associated angiosarcoma of approximately $0.1 \%$ [6]. These results are consistent with another older study, a series based on population studies in the Netherlands, which estimates an incidence of $0.16 \%$ [20].

Radiation-induced angiosarcoma generally affects the dermis within the radiation field: only the skin in $50 \%$ of cases, the skin and the breast in $40 \%$, and only in the breast parenchyma in $10 \%$ [21]. Our patient's angiosarcoma had an exclusively cutaneous presentation, specifically in the area of the surgical scar. In contrast, primary angiosarcoma usually originates from the breast parenchyma.

Secondary angiosarcoma is a rare neoplasm. Its clinical presentation is non-specific, with clinical signs that initially are usually subtle and appear benign; so they can be 
confused with inflammatory dermatoses induced exclusively by the effect of RT. This often involves a long time interval between the first symptom and the final diagnosis. Initially, it may simulate a bruise or it may present as purple-red colored macules, plaques or skin nodules. When it increased in size, ulcers or edema may appear [22]. Growth is insidious in low-grade angiosarcomas and explosive in high-grade tumors. It usually spreads hematogenously, so axillary lymph nodes are usually negative.

Contrary to what happens with lymphangiosarcoma (Stewart-Treves syndrome), the interval between RT and the development of angiosarcoma is short and lymphedema is minimal or absent. In a series with 27 radiation-induced breast angiosarcomas, the median interval was 59 months, and 5 of them occurred in less than 3 years [23]. In another series with 33 radio-induced breast angiosarcomas, the median interval between $\mathrm{BC}$ treatment and angiosarcoma was 74 months (range: 29-106) and appeared to decrease with age [20]. In our patient, the interval between RT and mammary angiosarcoma was 2.5 years (30 months).

Imaging also shows non-specific findings, which contributes to diagnostic delay [24]. Mammography may reveal a thickening of the skin and a poorly defined superficial mass. On ultrasound, they generally present as a heterogeneous hypervascular mass, associated with distortion of the breast architecture. Magnetic resonance imaging is more specific, typically showing a heterogeneous mass with low $\mathrm{T} 1$ and high $\mathrm{T} 2$ signals.

The definitive diagnosis is made by biopsy and histological study. Angiosarcoma is characterized by anastomosis of vascular channels lined by atypical endothelial cells. Ultrastructural features of the endothelium of blood vessels include well-formed junction complexes (zonula adherens type), a well-developed basal lamina, and Weibel- Palade bodies [25]. Histologically, angiosarcomas of the breast are classified according to the classification proposed by Donnell et al. [26]: grade I (well-differentiated: characterized by vascular channels invading the breast tissue with little endothelial proliferation), grade II (intermediately-differentiated: with scattered papillary endothelial formations and solid cellular foci) or grade III (poorly-differentiated: solid areas of spindle cells and papillary formations are prominent, additionally presents necrosis and hemorrhage).

Immunohistochemistry allows to identify the endothelial nature of the tumor and confirm the diagnosis. Many endothelial markers have been used, including: Factor VIII-related antigen, Ulex Europaeus lectin Agglutinin 1 (UEA-1), vascular endothelial growth factor (VEGF), PAL-E485, CD31 and CD34 [22,27]. The CD31 marker is considered the most sensitive and specific endothelial marker.

Due to the low frequency of radiation-associated breast angiosarcomas, there is no consensus on its therapeutic management.
Surgical resection with negative margins (R0) is the mainstay of treatment, via conserving surgery or mastectomy for breast lesions $[24,28]$. There is no clear consensus on the required margins. In general, a margin of $1 \mathrm{~cm}$ is accepted for small tumors and $3 \mathrm{~cm}$ for those with an infiltrative pattern [29]. Elective axillary lymphadenectomy is not justified, given the low frequency of regional lymph node metastases (11-20\%). The choice of adjuvant therapy is even more debatable. RT seems to produce additional benefit in these types of tumors; especially in high-grade tumors, large tumors and/or with positive or close

margins [30]. However, the role of adjuvant chemotherapy remains questionable; except in high-grade tumors, where it seems most beneficial, and in locally advanced cases (no capable of being surgically removed), where it constitutes the mainstay of treatment [30].

Angiosarcoma is an aggressive, easily infiltrative tumor, with high rate of local recurrence and metastasis. The most significant prognostic factor for recurrence is the size of the lesion at the time of diagnosis; therefore, early diagnosis is essential [31]. Local recurrences are frequent (45-60\%) despite extensive surgical removal of the tumor. Metastatic disease is generally preceded by local recurrence and the most common locations for metastasis are the lungs, the contralateral breast, and the bones [32]. Reported rates of advanced/metastatic disease at presentation range from 16 to $44 \%$, and overall median survival ranges from 6 to 16 months [32]. The 5-year survival rate is low, reported in the literature in the range of 28 to $69 \%[5,6,12,14,15,31,33,34]$. The patient in our case died 11 months after the diagnosis of angiosarcoma.

\section{Conclusion}

Radiation-induced breast angiosarcoma is a rare but very aggressive tumor, with high local recurrence rate and poor survival. Its diagnosis continues to be a challenge due to its non-specificity from the clinical, radiological and histological point of view. We must include this entity in the differential diagnosis of cutaneous lesions with vascular appearance, since it can be confused with inflammatory dermatoses induced by RT. Therefore, we must perform a biopsy of any suspicious skin lesion that occurs after RT, even in cases where the radiological evaluation does not coincide with the physical examination. Is essential to be able to make an early diagnosis and aggressive surgical treatment, since these are the most important prognostic factors of the disease. Breast angiosarcoma remains a clinical, radiological, and histological challenge; therefore a high index of suspicion in susceptible patients is required.

\section{Conflicts of Interest}

The authors declare that there is no conflict of interest regarding the publication of this paper. 


\section{Funding}

All authors have no source of funding

\section{Ethical Approval}

The patient of our report has given us her consent to publish all the information and images about her case.

\section{References}

1. McGowan TS, Cummings BJ, O Sullivan B, Catton CN, Miller N, et al. (2000) An analysis of 78 breast sarcoma patients without distant metastases at presentation. Int J Radiat Oncol Biol Phys. 46(2): 383-390.

2. Abdou Y, Elkhanany A, Attwood K, Ji W, Takabe K,et al. (2019) Primary and secondary breast angiosarcoma: single center report and a meta-analysis. Breast Cancer Res Treat. 178(3): 523-533.

3. Rodríguez Fernández V, Cameselle Cortizo L, Novo Domínguez A, Villar Fernández B, De Castro Parga G, et al. (2020) Angiosarcoma after radiotherapy for male breast cancer: A rare clinical case. Cancer Therapy 3:14

4. Fisher B, Anderson S, Bryant J, Margolese RG, Deutsch M, et al. (2002) Twenty-year follow-up of a randomized trial comparing total mastectomy, lumpectomy, and lumpectomy plus irradiation for the treatment of invasive breast cancer. N Engl J Med. 347(16): 1233-1241.

5. Kirova YM, Vilcoq JR, Asselain B, Sastre Garau X, Fourquet A. (2005) Radiation-induced sarcomas after radiotherapy for breast carcinoma: a large-scale single-institution review Cancer. 104(4): 856-863.

6. Rombouts AJM, Huising J, Hugen N, Siesling S, Poortmans PM, et al. (2019) Assessment of Radiotherapy-Associated Angiosarcoma After Breast Cancer Treatment in a Dutch Population-Based Study. JAMA Oncol. 5(2): 267-269.

7. Beck A. (1922) Zur Frage des Rontgenosarcomas Zugleich ein Beitrag zur Pathogenese des Sarcomas. Munch Med Wochenschr. 69: 623-625.

8. Warren S, Sommer GN. (1936) Fibrosarcoma of the soft parts with special reference to recurrence and metastasis. Arch Surg. 33: 425-450.

9. Cahan WG, Woodard HQ Higinbotham NL, Stewart FW, Coley BL. (1998) Sarcoma arising in irradiated bone: report of eleven cases. 1948. Cancer. 1998; 82(1): 8-34.

10. Wang L, Lao IW, Yu L, Yang W, Wang J. (2017) Primary Breast Angiosarcoma: A Retrospective Study of 36 Cases from a Single Chinese Medical Institute with Clinicopathologic and Radiologic Correlations. Breast J 2017; 23(3): 282-291.

11. Cohen Hallaleh RB, Smith HG, Smith RC, Stamp GF, Al Muderis O, et al. (2017) Radiation induced angiosarcoma of the breast: outcomes from a retrospective case series. Clin Sarcoma Res. 7: 15.

12. Salminen SH, Wiklund T, Sampo MM, Tarkkanen M, Pulliainen L, et al. (2020) Treatment and Prognosis of Radiation- Associated Breast Angiosarcoma in a Nationwide Population. Ann Surg Oncol 27(4): 1002-1010.

13. Arlen M, Higinbotham NL, Huvos AG, Marcove RC, Miller T, et al. (1971) Radiation- induced sarcoma of bone. Cancer. 28: 1087-1099.

14. Torres KE, Ravi V, Kin K, Yi M, Guadagnolo BA. et al (2013) Long-term outcomes in patients with radiation-associated angiosarcomas of the breast following surgery and radiotherapy for breast cancer. Ann Surg Oncol. 20(4): 1267-1274.

15. D’Angelo SP, Antonescu CR, Kuk D, Qin L, Moraco N, et al. (2013) Highrisk features in radiation-associated breast angiosarcomas. Br J Cancer 109: $2340-2346$.
16. Huang J, Mackillop WJ. (2001) Increased risk of soft tissue sarcoma after radiotherapy in women with breast carcinoma. Cancer. 92: 172-180.

17. Shah S, Rosa M. (2016) Radiation-associated angiosarcoma of the breast: clinical and pathologic features. Arch Pathol Lab Med. 140(5): 477-481.

18. Sheth GR, Cranmer LD, Smith BD, Grasso Lebeau L, Lang JE. (2012) Radiation-induced sarcoma of the breast: a systematic review. Oncologist. 2012;17(3): 405-418.

19. Rubino C, Shamsaldin A, Lê MG, Labbé M, Guinebretière JM,et al. (2005) Radiation dose and risk of soft tissue and bone sarcoma after breast cancer treatment. Breast Cancer Res Treat. 89(3): 277-288.

20. Strobbe LJ, Peterse HL, Van Tinteren H, Wijnmaalen A, Rutgers EJ. (1998) Angiosarcoma of the breast after conservation therapy for invasive cancer, the incidence and outcome. An unforseen sequela. Breast Cancer Res Treat. 47(2): 101-109.

21. Majeski J, Austin RM, Fitzgerald RH. (2000) Cutaneous angiosarcoma in an irradiated breast after breast conservation therapy for cancer: association with chronic breast lymphedema. J Surg Oncol. 74(3): 208-212.

22. Zemanova M, Machalekova K, Sandorova M, Boljesikova E, Skultetyova M, et al. (2013) Clinical management of secondary angiosarcoma after breast conservation therapy. Rep Pract Oncol Radiother 19(1): 37-46.

23. Billings SD, McKenney JK, Folpe AL, Hardacre MC, Weiss SW. et al (2004) Cutaneous angiosarcoma following breast-conserving surgery and radiation: an analysis of 27 cases. Am J Surg Pathol. 28(6): 781-788.

24. Toesca A, Spitaleri G, De Pas T, Botteri E, Gentilini O, et al. (2012) Sarcoma of the breast: outcome and reconstructive options. Clin Breast Cancer. 12(6): 438-444.

25. Carstens PH. (1981) The Weibel-Palade Body in the diagnosis of endothelial tumors. Ultrastruct Pathol. 2(4): 315-325.

26. Donnell RM, Rosen PP, Lieberman PH, Kaufman RJ, Kay S, et al. (1981) Angiosarcoma and other vascular tumors of the breast. Am J Surg Pathol. 5(7): 629-642.

27. Taghipour Zahir S, Sefidrokh Sharahjin N, Rahmani K. (2014) Primary breast angiosarcoma: pathological and radiological diagnosis. Malays J Med Sci. 21(5): 66-70.

28. Amajoud Z, Vertongen AS, Weytens R, Hauspy J. (2018) Radiation induced angiosarcoma of the breast: case series and review of the literature. Facts Views Vis Obgyn. 10(4): 215-220.

29. Al-Benna S, Poggemann K, Steinau HU, Steinstraesser L. (2010) Diagnosis and management of primary breast sarcoma. Breast Cancer Res Treat 2010;122(3): 619-626.

30. Cao J, Wang J, He C, Fang M. (2019) Angiosarcoma: a review of diagnosis and current treatment. Am J Cancer Res. 9(11): 2303-2313.

31. Depla AL, Scharloo-Karels CH, De Jong MAA, Oldenborg S, Kolff MW, et al. (2014) Treatment and prognostic factors of radiation-associated angiosarcoma (RAAS) after primary breast cancer: a systematic review. Eur J Cancer. 50(10): 1779-1788.

32. Abbott R, Palmieri C. (2008) Angiosarcoma of the breast following surgery and radiotherapy for breast cancer. Nat Clin Pract Oncol. 5(12): 727-736.

33. Buehler D. (2014) Angiosarcoma outcomes and prognostic factors. Am J Clin Oncol. 37:473-479.

34. Vorburger SA, Xing Y, Hunt KK, Lakin GE, Benjamin RS, et al. (2005) Angiosarcoma of the breast. Cancer. 104(12): 2682-2688. 